\title{
Experiments and Numerical Simulation of Flow Field Inner the Chain Plate Fiber Dryer
}

\author{
Li Hui and Wang Rui-li \\ Huang He science and Technology University \\ 549138183@qq.com;63876193@qq.com
}

\begin{abstract}
The numerical calculation model was founded on partial data of the actual test of flow field in chain plate fiber dryer, then the computational fluid dynamics (CFD) software Fluent was adopted to analyze the numerical result of dryer box unit. The result show: the numerical calculation method and data were reliable; the flow relation formula was obtained by air flow experiment in which air flew through fiber layer and chain plate, could be treated as additional momentum source term equation of air through the porous media; owing to the unreasonable setting of open porosity of air distribution plate in the dryer, there was obviously non-uniform air distribution above fiber layer, which would lead to non-uniform drying phenomenon; because of the centrifugal force of fan and diversion effect of volute, there was obvious velocity component in y direction, which could enhance the convective mass transfer effect. The research result could become the basis of design, on which the construction of chain plate fiber dryer was optimized, especially in the optimization of air distribution plate and volute of fan.
\end{abstract}

Keywords: Chain plate fiber dryer ; Numerical simulation ; Porous media ;Optimization

\section{Introduction}

During the production processes of a variety of man-made fibers (acrylic, polyester, viscose, etc.), a chain plate fiber dryer was often adopted as a drying and heat-setting post-process equipment. The chain plate fiber dryer is a kind of belt dryer, which was of the easy operation and simple structure. It was estimated that $0.25 \mathrm{~kg}$ of coal was consumed when obtaining $1 \mathrm{~kg}$ of dried fiber in the drying process. Therefore, studying the gas flow characteristics inside the chain plate fiber dryer, optimizing the structure and reducing the energy consumption were necessary and significant to realize the energy savings of the textile industry.

The technology on the chain plate dryer in China was introduced from abroad by Zhengzhou Textile Machinery Co., Ltd during the period of "Eighth Five-Year". Relevant experts studied the operating characteristics of the dryer just on the optimal combination of different types of dryer [1], yet the systematic research on the inner field of the chain plate dryer hadn't been done to achieve the chain plate dryer's performance optimization.

Due to the huge dryer volume and poor working conditions in actual operation, using conventional testing methods can not fully grasp the flow field characteristics. Therefore, the technology of computational fluid dynamics (CFD) became an important tool for studying the inner flow of the dryer. Through the numerical simulation on a type of fruit tray dryer, Mathioulakis E. et al. compared simulation results with the experiment results and obtained the relationship between the rate of drying and the air 
flow [2]. Yue Xiaoyu, etc. proposed a set of evaluation criteria based on the results obtained from the numerical simulation of a multi- belt dryer [3]. Wu Yang proposed improve the uniformity of the flow field by the distributing plate based on the results obtained from the numerical simulation of a belt dryer for drying cellulose acetate [4]. Wang He-shi got the flow distribution of the honeycomb dryer by the method of numerical simulation [5]. Although the basic working principles of the chain plate dryer is similar with the dryer above, the research on its field flow is still blank because of the existence of the air distribution plate, fiber layers, chain boards and other porous medium area, as well as strong turbulence generated within the circulating fan.

In this paper, the numerical calculation model of chain plate dryer box unit was founded on taking full account of the operating conditions characteristics of chain plate dryer. The simulation results were compared with the actual test results and the characteristics of flow field inside the chain plate dryer was analyzed, which provided a reference for the performance optimization of chain plate fiber dryer.

\section{The Structure of Chain Plate Dryer and Actual Test}

\subsection{The Structure of Chain Plate Dryer and Operating Conditions Characteristics}

The prototype chosen for testing was ZHR594A chain plate dryer and its general diagram was shown in Figure 1 (a). The capacity of this type of dryer was $3.2 \mathrm{t} / \mathrm{h}$ (dry basis) and it consisted of 14 box units. The length $\times$ width $\times$ height of every box unit was $2184 \mathrm{~mm} \times 4460 \mathrm{~mm} \times 2623 \mathrm{~mm}$. Each box was connective through the air distribution plate and chain plate. Fiber material evenly was laid on the chain plate surface by the feeding device and then was conveyed into the drying room by the chain. The air blown by the fan was heated through the heater and then went through the material layer on the air distribution plate. Moisture was evaporated quickly into the hot air. Part of hot and humid air was drawn out of the dryer by the fan and most of them went into circulation in order to achieve the purpose of drying. The inner structure of the dryer was shown in Figure 1 (b). The outer rim diameter of the impeller of circulating fan was $759 \mathrm{~mm}$ and the inner rim diameter was $604 \mathrm{~mm}$ and the outlet width was $235 \mathrm{~mm}$. The backward straight vane was chosen and its outlet installation angle was $59^{\circ}$.The rotational speed of fan was $1000 \mathrm{rpm}$ under the design operating conditions. The inner room of the dryer was divided into three equal parts by three air distribution plates which were respectively $4.78 \%, 9.35 \%, 14.8 \%$ open porosity (pore diameter $3 \mathrm{~mm})$.

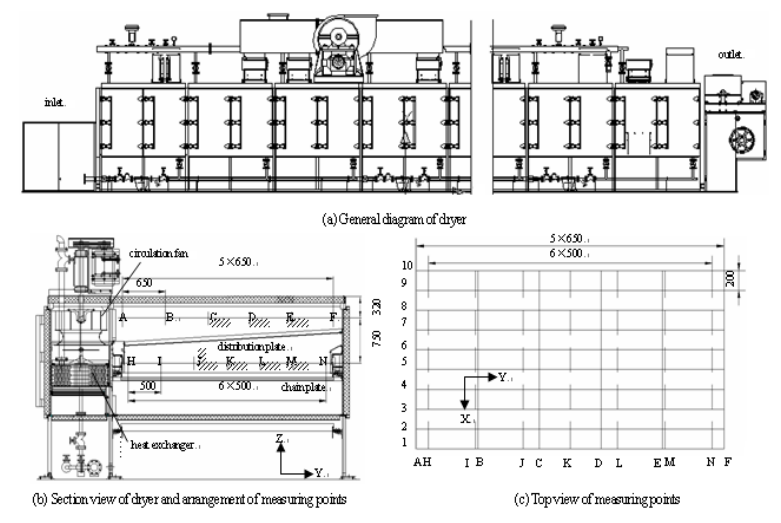

Figure 1. Schematic Diagram of Chain Plate Fiber Dryer 


\subsection{Actual Cold Test}

Figure 1 (b) was a cross-sectional view of dryer box unit and showed the distribution of measuring points. Chain moved away the $\mathrm{X}$-axis positive direction shown in Figure 1. The horizontal plane $320 \mathrm{~mm}$ away from surface of the dryer box outer shell and $110 \mathrm{~mm}$ height away from the chain plate was the test area.. Measuring point distribution was shown in Figure 1 (c). There were 10 rows of measuring points arranged in the longitudinal direction at intervals of $200 \mathrm{~mm}$ of the length of each chain plate. 6 columns measuring points were arranged upper of the air distribution plate and 7 columns measuring points were arranged upper of the fiber layer. Dashed line in Figure 1 stood for measuring points upper of the fiber layer. The average speed of each row and each column can be computed on the basis of the air flow velocity of every measuring point obtained from the actual cold test. Standard deviation was compared to analyze the degree of nonuniform of the inner flow field in dryer.

On the premise of closing the generality, $100 \mathrm{~mm}$ thick cotton fiber layer was laid on the chain plate instead of the synthetic fibers. Because of the consistency of every box unit operating condition, only a box unit was tested when the chain plate dryer worked. KA22type hot bulb anemometer was adopted to test the velocity of airflow.

\subsection{Resistance Test of Cotton Fiber Layer}

Cotton fiber layer and the chain plate can be considered as porous medium. So, the flow of air through the chain plates and the cotton fiber layer can be considered as the flow of fluid through a porous medium. Darcy's law which is used to describe fluid flow through porous media is only applicable to $\operatorname{Re}<3$ linear layer flow. So, it is necessary to increase additional items on the base of Darcy classic formula. Inertial effects items were usually added to the macro expression of Darcy's law in order to get new flow relationship [6]:

$$
|\Delta P|=\frac{\mu}{\alpha} v \Delta n+C_{2} \frac{1}{2} \rho v^{2} \Delta n
$$

wherein, $\alpha$ was the permeability and $\mu$ was the viscosity coefficient and $C_{2}$ was the coefficient relating to the fluid and porous medium. The first term is an expression of Darcy's law in (1) and the second term is the inertial loss term.

Air flow method was adopted to test the relationship between fiber layer and flow velocity, and the experimental apparatus was shown in Figure 2.When test began, the compressed air from air compressor tank was conveyed to a $80 \mathrm{~mm}$ inner diameter and $2 \mathrm{~m}$ long plastic tube after reduced to standard atmosphere by pressure maintaining valve and adjusted rate of the air flow. The pore plate which was the same specifications with chain plate was arranged $1.4 \mathrm{~m}$ away from the inlet of the tube and the $100 \mathrm{~mm}$ thick layer of cotton fiber was laid on the pore plate. The velocity of air flow was tested by the anemometer and the resistance (total pressure) loss through the fiber layer and pore plate was tested by microtasimeter. The cotton used in the experiment was consistent with the actual test sample and its density was $0.0084 \mathrm{~g} / \mathrm{cm}^{3}$. The test results and the fitted curve were shown in Figure 3. 


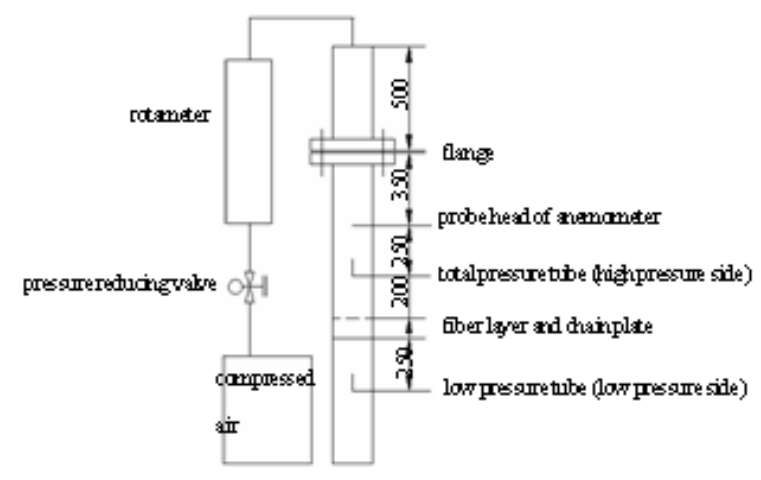

Figure 2. Schematic Diagram of Measuring Equipment of Resistance of Cotton Fiber Layer

As shown in Figure 3, when the velocity of air flow was in the range of $0.46 \mathrm{~m} / \mathrm{s} \sim 2.65 \mathrm{~m} /$ $\mathrm{s}$, the relationship between the resistance (total pressure) loss and velocity can be represented by formula (2).

$$
\Delta P=81.7712 v^{2}+18.2035 v \quad R^{2}=0.9979
$$

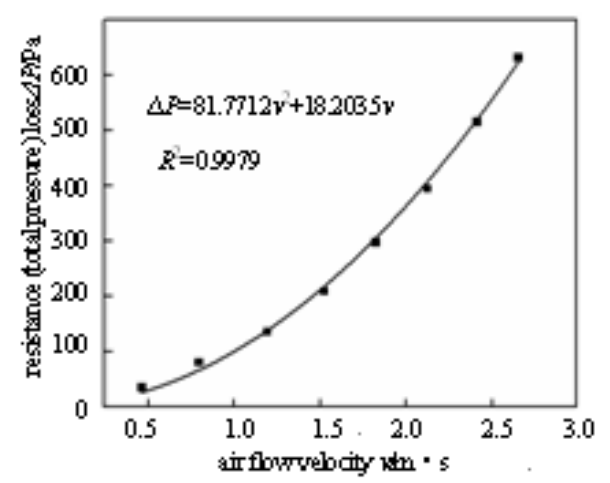

Figure 3. "Resistance Loss-velocity" Relation Curve of Air Flow through the Fiber Layer

\section{Establishment of Computational Fluid Dynamics Model}

\subsection{Model Simplification and Mesh}

Due to the consistency of every box structure of chain plate dryer, only one box unit need to be simulated. The connecting area of each box can be regarded as a periodic plane and the influence of transmission components (chain plate, chain) inside the box was ignored.

The aim of investigation was the air flow distribution of stable working condition, so the simulation can be carried out in steady-state condition. Gambit which was the preprocessing software of fluent was adopted to mesh the geometric model. Considering the stronger turbulence near the fan, the tetrahedral grid was adopted to have a local encryption. The rest of the area was used the structured grid. At last, the total number of the grids was about 1.8 million, as shown in Fig.4. 
Figure 4. Mesh Diagram of Box Unit

\subsection{Turbulence Model and Boundary Conditions}

Standard k- $\varepsilon$ turbulence model which was the fully developed turbulence was adopted. The standard wall function was used near the wall. Governing functions included continuity equation, momentum equation, energy equation, k equation and eequation [7]:

$$
\begin{gathered}
\rho \frac{\partial k}{\partial t}+\rho u_{j} \frac{\partial k}{\partial x_{j}}=\frac{\partial}{\partial x_{j}}\left[\left(\mu+\frac{\mu_{t}}{\sigma_{k}}\right) \frac{\partial k}{\partial x_{j}}\right]+\mu_{t} \frac{\partial u_{i}}{\partial x_{j}}\left(\frac{\partial u_{i}}{\partial x_{j}}+\frac{\partial u_{j}}{\partial x_{i}}\right)-\rho \varepsilon \\
\rho \frac{\partial \varepsilon}{\partial t}+\rho u_{k} \frac{\partial \varepsilon}{\partial x_{k}}=\frac{\partial}{\partial x_{k}}\left[\left(\mu+\frac{\mu_{t}}{\sigma_{\varepsilon}}\right) \frac{\partial \varepsilon}{\partial x_{k}}\right]+\frac{c_{1} \varepsilon}{K} \mu_{t} \frac{\partial u_{i}}{\partial x_{j}}\left(\frac{\partial u_{i}}{\partial x_{j}}+\frac{\partial u_{j}}{\partial x_{i}}\right)-c_{2} \rho \frac{\varepsilon^{2}}{K}(4) \\
\mu_{t}=\frac{c_{\mu} \rho k^{2}}{\varepsilon}
\end{gathered}
$$

where, $\mathrm{c}_{\mathrm{u}}=0.09, \quad \mathrm{c}_{1}=1.44, \quad \mathrm{c}_{2}=1.92, \quad \sigma_{\mathrm{k}}=1.0, \quad \sigma_{\varepsilon}=1.3$

Because the entire flow field was seen steady-state flow, MRF (Moving Reference Frame) model was adopted for the simulation of the rotating part of the internal circulation fan. MRF (Moving Reference Frame) model was approximate method for solving the unsteady problem of operation and the rotational speed of fan was 1000rpm.

The area of cotton fiber layer, chain plate and air distribution plate was continuous and can be seen as the boundary conditions of porous media. The essence was to add an additional momentum loss term in the source term of momentum equation. The governing equations of porous media model consisted of two parts: one part was viscous loss term; the other part was the inertial loss term, namely:

$$
S=-\left(\sum_{j=1}^{3} D_{i j} u v_{j}+\sum_{j=1}^{3} C_{i j} \frac{1}{2} \rho|v| v_{j}\right)
$$

for the simple isotropic porous medium, formula (6) can be simplified as:

$$
\begin{gathered}
S_{i}=-\left(\frac{\mu}{\alpha} v_{i}+C_{2} \frac{1}{2} \rho\left|v_{i}\right| v_{i}\right) \\
\Delta P=-S_{i} \Delta n
\end{gathered}
$$

where, $\alpha$ is the permeability, C2 was inertial drag factor ${ }^{[8]}$.

Comparing formula (1) with formula (7), we can easily get the expression of $\alpha$ and $\mathrm{C} 2$. The cotton fiber layer and the chain plate were seen as the same area in experiment and the flow relationship between cotton fiber layer and the chain plate was integrated 
into the equation (2). Therefore, from equation (2), the viscous drag factor and inertial resistance factor can be obtained respectively as: $1 / \alpha=1.017 \times 107, \mathrm{C} 2=1335.4$.

Air distribution plate was a perforated thin flat plate and its area was much larger than the thickness. Therefore, the viscous loss term in the additional momentum source term was ignored in the numerical model and the flow relationship was as follows ${ }^{[8]}$ :

$$
|\Delta P|=C_{2} \frac{1}{2} \rho v^{2} \Delta n
$$

according to the experimental formula obtained by Smith and Van Winkle et al ${ }^{[9]}$ :

$$
\stackrel{\&}{m}=C A_{f} \sqrt{(2 \rho \Delta p) /\left(1-\left(A_{f} / A_{p}\right)^{2}\right)}
$$

where, $A_{p}$ was the total area of pore plate; $A_{f}$ was the area of opening pore; $C$ was the coefficient relating to Reynolds number, diameter of pore and thickness of plate. The mass flow through the pore plate can be obtained by reference [6] as $\stackrel{\&}{m}=\rho v A_{p}$.Comparing (8) with (9), we can get:

$$
C_{2}=\frac{1}{C^{2}} \frac{\left(A_{p} / A_{f}\right)^{2}-1}{\Delta n}
$$

In the chain plate dryer, the opening rate of air distribution plate increased from near the fan to away the fan. The air distribution plate was divided into three parts and the opening rate of the three parts was respectively $4.78 \%, 9.35 \%, 14.8 \%$. Mass flow can be computed by the average measured velocity of different opening rate pore plate $(3.85 \mathrm{~m} / \mathrm{s} 、 4.19 \mathrm{~m} / \mathrm{s} 、 2.31 \mathrm{~m} / \mathrm{s})$ to obtain three different $\mathrm{C} 2: 513100 、 136100$ 、 49170. Compared with the overall size of chain plate dryer, the fins of the heater which were used to enhance the heat exchange were very small. So, the influence of the fins was ignored during the numerical simulation. Considering the resistance loss of the air flow through the heater, the area of heater was seen as porous in the process of numerical simulation and the resistance loss can be solved by momentum equation of porous medium. When setting boundary conditions in fluent software, momentum source equation in the area of porous medium can be represented not only by a quadratic function equation (6), but also by a power function (12):

$$
S_{i}=-C_{0}|v|^{C_{1}-1}
$$

From the reference [10], the friction coefficient and pressure loss when air flowed through the fin-tube heat exchanger can be calculated by formula (13) and (14):

$$
\begin{gathered}
f=37.86\left(\frac{G_{\max } d_{r}}{\mu}\right)^{-0.316}\left(\frac{S_{1}}{d_{r}}\right)^{-0.927}\left(\frac{S_{1}}{S_{2}}\right)^{0.515} \\
\Delta P=f \frac{n G_{\max }^{2}}{2 \rho}
\end{gathered}
$$

where, $\mathrm{G}_{\max }$ was the average mass flow through the narrowest flow cross section; $\mathrm{S} 1$ and $S 2$ were respectively horizontal pitch and vertical pitch; $d_{r}$ was the diameter of root of fin; $n$ was the number of rows of tubes.Combining equation (13) with equation (14) and converting $\mathrm{G}_{\max }$ to $\mathrm{v}_{\max }$, we can get:

$$
\Delta P=18.93 n\left(\frac{d_{r}}{\mu}\right)^{-0.316}\left(\frac{S_{1}}{d_{r}}\right)^{-0.927}\left(\frac{S_{1}}{S_{2}}\right)^{0.515} \rho^{0.684} v_{\max }^{1.684}
$$


where, $v_{\max }$ was the actual velocity of the air flow through the smallest cross-section and $v_{\max }$ must be converted to the apparent velocity in Fluent. So, the equation (15) can be represented as:

$$
\Delta P=18.93 n\left(\frac{d_{r}}{\mu}\right)^{-0.316}\left(\frac{S_{1}}{d_{r}}\right)^{-0.927}\left(\frac{S_{1}}{S_{2}}\right)^{0.515} \rho^{0.684}\left(v \times \frac{A_{\max }}{A_{\min }}\right)^{1.684}
$$

In equation (16), $\mathrm{A}_{\max }$ was the area of the largest circulation cross-section and $\mathrm{A}_{\min }$ was the area of the smallest circulation section. The diameter of parent tube of fin on the heater was $22 \mathrm{~mm}$; the height of fin was $16 \mathrm{~mm}$; the length of a single tube was 1816 $\mathrm{mm}$. In actual test, $\mathrm{G}_{\max }=23.46 \mathrm{~kg} /\left(\mathrm{m}^{2} * \mathrm{~s}\right)$. Integrating (12), (13), (14), (16), we obtained $\mathrm{C}_{0}=30.374 、 \mathrm{C}_{1}=1.684$.

Implicit uncoupled solver was used to calculate governing equations and secondorder upwind was used to discrete the convection term. The algorithm of SIMPLE was adopted to solve the field and the convergence precision was of $10^{-4}$.

\section{Comparison and Analysis of Results}

\subsection{Flow Field Upper the Air Distribution Plate}

The actual air flow velocity of testing points was shown in Figure 5 and the simulation result was shown in Figure 6. Figure 7 was the numerical simulation contours. As the role of centrifugal force of flow, the velocity distribution of the air flow was not uniform. As shown in Figure 7, because of the centrifugal action of the circulation fan and round volute, a large part of the gas would flow away from the outlet of the fan at the speed of $10 \mathrm{~m} / \mathrm{s}$ or more down the fan volute. A region of high flow velocity was formed along tangential of the outlet in quite a distance.



Figure 5. The Measured Results of Air Flow Velocity in the Upper Area of Air Distribution Plate

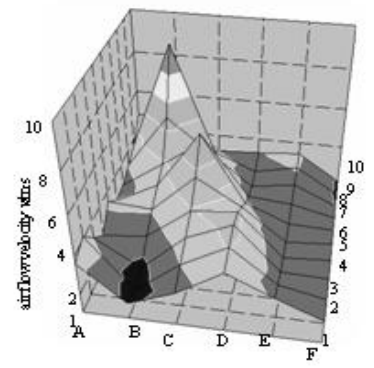

Figure 6. The Numerical Results of Air Flow Velocity in the Upper Area of Air Distribution Plate 




\section{Figure 7. Contours of Air Flow Velocity in the Upper Area of Air Distribution Plate in the Numerical Simulation}

Table 1 and Table 2 were respectively testing mean value and standard deviation of testing results and numerical simulation results. Comparing Table 1 with Table 2, we can obtain the conclusions that the average velocity of testing point $A$, point $B$ and point $\mathrm{C}$ near the fan was greater than that of testing point $\mathrm{D}$, point $\mathrm{E}$ and point $\mathrm{F}$. But the greater the velocity was, the more non-uniform the flow field was.

Air distribution plate played an important role for drying effect and its opening pore rate would directly affect the uniformity of drying process. As the results of numerical simulation, the air mass flux through the air distribution plate of $4.78 \%, 9.35 \%, 14.8 \%$ opening pore rate was respectively $0.94 \mathrm{~kg} / \mathrm{s} 、 1.58 \mathrm{~kg} / \mathrm{s} 、 2.37 \mathrm{~kg} / \mathrm{s}$. Where the air mass flux was small, the effect of drying was inevitable bad. So, the non-uniformity of the air mass flux through the chain plate would inevitable lead to the non-uniform of drying effect, which was consistent with the non-uniform phenomenon in practical production.

Table 1. The Average Velocity and Standard Deviation in the Upper Area of Air Distribution Plate in the Actual Test

\begin{tabular}{|c|c|c|c|c|c|c|c|c|c|c|c|c|c|c|c|c|}
\hline \multicolumn{17}{|c|}{ Measuring points } \\
\hline & $A$ & $B$ & $C$ & $D$ & $E$ & $F$ & 1 & 2 & 3 & 4 & 5 & 6 & 7 & 8 & 9 & 10 \\
\hline $\begin{array}{c}\bar{v} \\
/(\mathrm{m} / \mathrm{s})\end{array}$ & 3.50 & 4.19 & 5.15 & 3.23 & 2.69 & 1.93 & 2.13 & 2.48 & 2.78 & 2.47 & 2.87 & 3.58 & 3.93 & 4.03 & 4.32 & 5.88 \\
\hline$R$ & 1.00 & 2.64 & 2.69 & 1.58 & 0.96 & 0.73 & 0.46 & 0.86 & 1.19 & 0.93 & 0.95 & 0.49 & 1.64 & 2.19 & 2.75 & 3.72 \\
\hline
\end{tabular}

Table 2 The average velocity and standard deviation in the upper area of air distribution plate in the numerical simulation

\begin{tabular}{|c|c|c|c|c|c|c|c|c|c|c|c|c|c|c|c|c|}
\hline \multicolumn{17}{|c|}{ Measuring points } \\
\hline & $A$ & $B$ & $C$ & $D$ & $E$ & $F$ & 1 & 2 & 3 & 4 & 5 & 6 & 7 & 8 & 9 & 10 \\
\hline $\begin{array}{c}\bar{v} \\
/(\mathrm{m} / \mathrm{s})\end{array}$ & 3.43 & 3.91 & 4.29 & 3.69 & 2.81 & 2.28 & 2.92 & 3.09 & 3.07 & 3.14 & 3.48 & 3.59 & 3.46 & 3.60 & 4.12 & 3.53 \\
\hline$R$ & 0.68 & 2.29 & 1.26 & 0.74 & 0.39 & 0.19 & 0.85 & 1.08 & 1.03 & 0.99 & 1.07 & 0.62 & 1.28 & 1.39 & 2.49 & 1.04 \\
\hline
\end{tabular}

\subsection{Flow Field Upper the Fiber Layer}

Figure 8 and Figure 9 were respectively the testing result and numerical simulation result upper the fiber layer. Table 3 and table 4 were respectively the average velocity and standard deviation of air flow upper the fiber layer obtained by actual test and numerical simulation. 
The air mass flux through the air distribution plate of different opening pore rate was respectively $0.94 \mathrm{~kg} / \mathrm{s}, 1.58 \mathrm{~kg} / \mathrm{s}, 2.37 \mathrm{~kg} / \mathrm{s}$ and the pressure loss was respectively $71.8 \mathrm{~Pa}$, $49.31 \mathrm{~Pa}$, and $39.02 \mathrm{~Pa}$. The air distribution plate of $4.78 \%$ opening pore rate had the smallest mass flux and the largest pressure loss. Comparing Fig.8 and Fig.9, we can see that the area of lower air velocity and larger standard deviation corresponded to the area of air distribution plate of the smallest opening pore rate. So, we can draw the conclusion that the key factors leading to non-uniform air distribution were the irrational opening pore of air distribution plate.

Comparing Table 3 and Table 4, we can see that the standard deviation of horizontal measuring point was larger than that of the longitudinal measuring point, which indicated that the non-uniformity of the flow field was larger in perpendicular to the movement direction of the chain plate. The non-uniformity of the field flow in the direction of movement of chain plate can be improved and even eliminated when the material went through different box unit of dryer with the chain plate. But the nonuniformity of the flow field in perpendicular to the movement direction of the chain plate can not be improved. So, changing the structure design and especially changing the distribution of the opening pore rate should be done to improve the non-uniformity of the air distribution in perpendicular to the movement direction of the chain plate.

Comparing the tested results with the numerical simulation results, we can see that the simulation results agreed well with the tested results. So, the method of numerical simulation can be used to analyze and predict the flow characteristics of air flow in the chain plate dryer, and then to optimize the structure of the dryer and improve the drying efficiency and save energy.

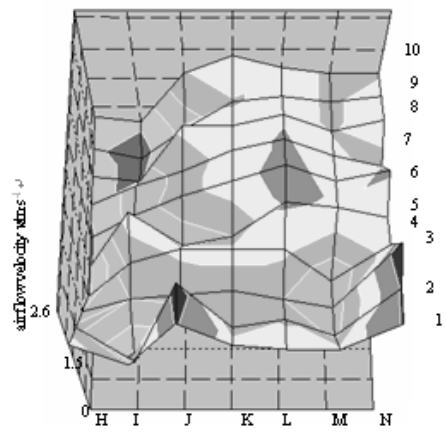

Figure 8. The Measured Results of Air Flow Velocity in the Upper Area of Fiber Layer



Figure 9. The Numerical Results of Air Flow Velocity in the Upper Area of Fiber Layer 
Table 3. The Average Velocity and Standard Deviation in the Upper Area of Fiber Layer in the Actual Test

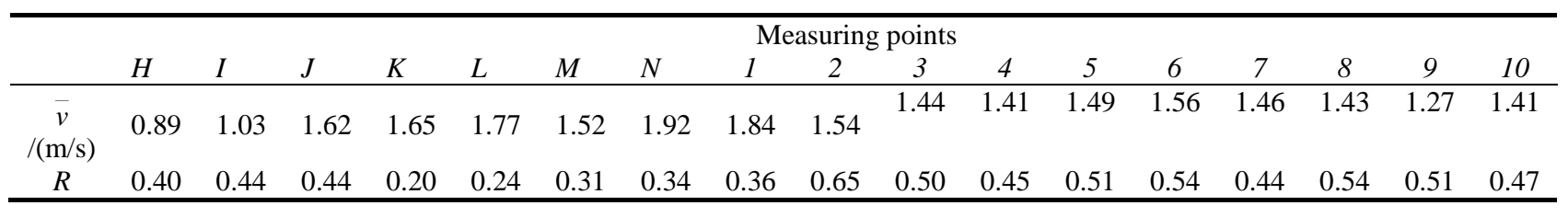

Table 4 The average velocity and standard deviation in the upper area of fiber layer in the numerical simulation

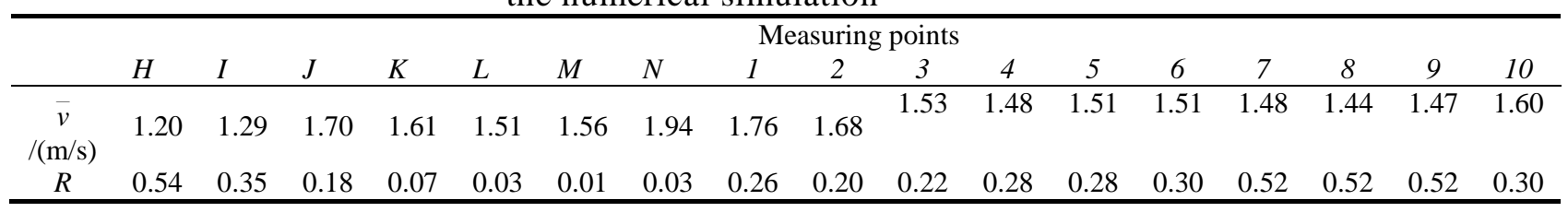

\subsection{Overall Analysis of the Air Flow Field in the Dryer}

The trace lines of air field flow in dryer box unit were shown in Figures 10-12 showed the trace lines of field respectively on the plane of $y=0$ and $y=-1$. Figures $10-12$ showed that due to no partition between the adjacent box unit and the centrifugal force at the fan outlet and the diversion effect of the volute, obvious positive $y$-axis velocity $\left(\mathrm{v}_{\mathrm{y}}=0.78 \mathrm{~m} / \mathrm{s}\right)$ component existed in the flow field of dryer box unit. The existence of velocity component can enhance the convective mass transfer effects in the process of drying, which was different with general belt dryer. As shown in Figure 13, when air flew through the air distribution plate and fiber layer, the resistance (total pressure) loss would produce. It can be calculated that the resistance loss absorbed by the air distribution plate was 44Pa; the resistance loss absorbed by the fiber layer and chain plate was 37Pa; the resistance loss through the heater was 56Pa.



Figure 10. Path Line of Box Unit of Dryer

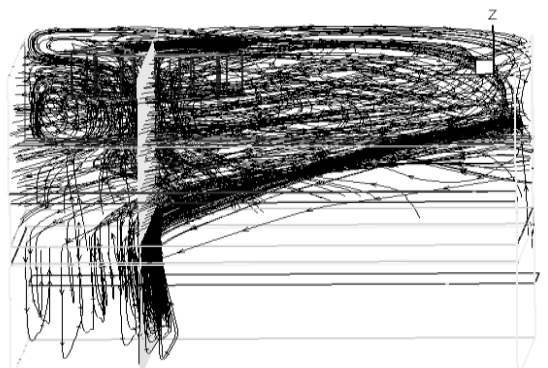

Figure 11. Path Line through Plane of $y=0 \mathrm{~m}$ 


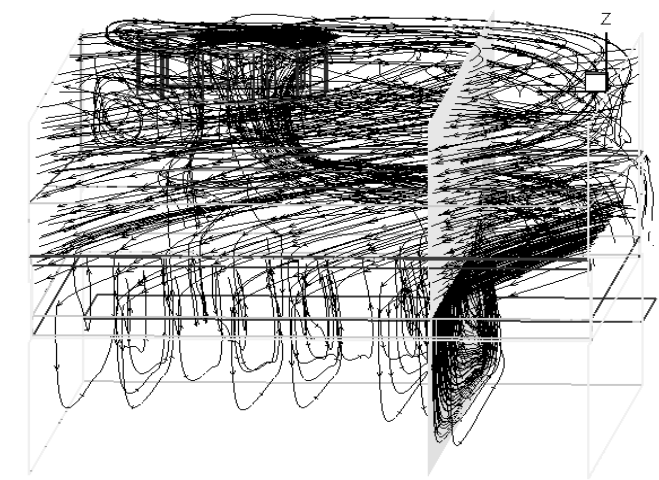

Figure 12. Path Line through Plane of $y=-1 m$

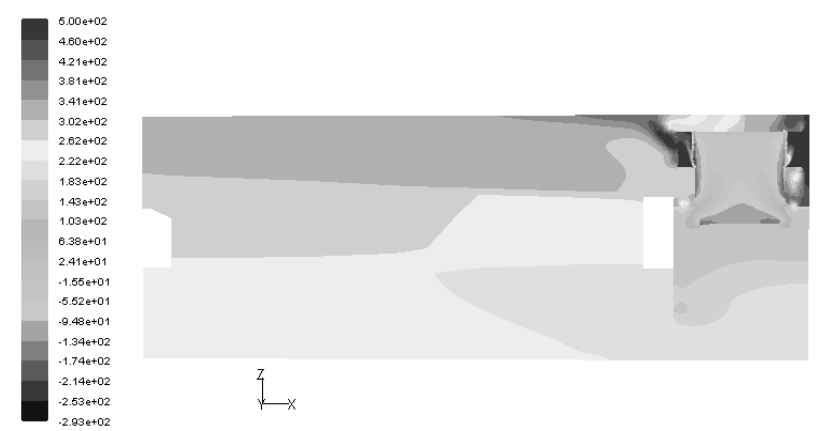

Figure 13. Contours of Total Pressure of Plane of $y=0 m$

\section{Conclusions}

On the basis of the part tested data on the air flow field in the chain plate fiber dryer, the model aiming the chain plate dryer founded on the numerical calculation and the numerical simulation results obtained by fluid dynamics software Fluent were reliable. The method of CFD (computational fluid dynamics) can be used to analyze the air flow field inner the chain plate dryer and to have a optimal design for the structure and to provide the basis for energy savings. Comparing tested results with simulation results, the conclusion can be drawn as follows:

1) The flow relationship formula obtained by the air flow experiment that the air flew through the fiber layer and chain plate can be used as the additional energy source term formula when the air in the chain plate fiber dryer flew through the porous media. The flow loss through the heat exchanger can also be obtained by simplifying the model and using the porous media model

2) The opening pore rate of studied air distribution plate was unreasonable, which made the non-uniform distribution of air flow produce in the direction of perpendicular to movement of the chain plate. The non-uniformity of the air distribution would lead to the non-uniformity of drying effect. So, it was necessary to discuss the problem of uniform air distribution.

3) Due to the centrifugal force of the fan and diversion effect of the volute, the obvious positive $y$-axis velocity component existed in the flow field of chain plate dryer. The velocity component was helpful to enhance the convective mass transfer effects in the process of drying. 


\section{Symbol Description}



\section{References}

[1] W. Zhu and B. Wu, "Rotary screen dryer and chain plate dryer's optimization optimal grouping", Proceedings of national drying conference, (2002) March 13-15; Harbin, China.

[2] E. Mathioulakis, V. T. Karathanos and V. G. Belessiotis, "Journal of Food Engineering", vol. 5, no. 36, (1998).

[3] X. Yue, J. Zhao and E. Shi, “Asia-Pac. J. Chem. Eng.”, vol. 2, no. 12, (2007).

[4] Y. Wu, "Study on the kinetics of cellulose acetate drying and CFD simulation for the dryer", Tsinghua University Press, Beijing (2006).

[5] H. Wang, "Honeycomb cardboard drying engineering research", Tsinghua University Press, Beijing (2007).

[6] R. Lin, "Introduction of heat and mass transfer in porous media", Science Press, Beijing (2005).

[7] F. Wang, "Analysis of computational fluid dynamics-the principle and application of CFD software", Tsinghua University Press, Beijing (2004).

[8] Z. Han, "Fluent 6.3 user's guide", Tsinghua University Press, Beijing (2009).

[9] P. L. Smith and M. V. Winkle, "AIChE Journal", vol. 4, no. 26, (1998).

[10] Q. Songwen, "Design handbook of heat exchanger", Chemical Industry Press, Beijing (2002).



\section{Author}

\section{Li Hui}

